DOI https://doi.org/10.30525/978-9934-588-81-5-1.10

\title{
SALMONELLA-INDUCED CHANGES IN THE GUT MICROBIOME
}

\author{
Bukina Iu. V. \\ Assistant at the Department of Microbiology, Virology and Immunology \\ Zaporizhzhia State Medical University \\ Polishchuk N. M. \\ Ph.D., \\ Associate Professor at the Department of Microbiology, \\ Virology and Immunology \\ Zaporizhzhia State Medical University \\ Zaporizhzhia, Ukraine
}

The intestinal microbiome significantly affects the functioning of the body: it participates in metabolic processes, inhibition of pro-inflammatory reactions, in the formation of innate and adaptive immune response in the intestinal mucosa [1, 478-485; 2, 1131-1141; 3, 327-336; 4, 107-114]. The most important function of the intestinal microbiome is to protect the body from pathogenic microorganisms - pathogens of bacterial intestinal infections [5, 409-414; 6, 82-91]. It is known that dysbiotic changes in the intestine lead to increased susceptibility to pathogenic bacteria, such as salmonella [7, 231-241; 8, 3216-3223], which are the etiological factor of gastroenteritis [9, 459-466]. One of the most common causes of microbiota changes is the use of antibiotics [10, 201-208; 11, 621-626; 12, 138-145]. Therefore, of particular interest are the processes of interaction of antibiotics, Salmonella enteritidis and Salmonella typhimurium with representatives of the normal intestinal microflora [13, 435-454; 14, 1536-1545; 15, 1091-1093]. The aim to analyze changes in the quantitative and species composition of the small intestine microbiota in rats with salmonellainduced bowel inflammation on the background of vancomycin and $B$. fragilis administration. Methods. All rats, except group I (control, intact), received vancomycin and/or suspension of microorganisms. In order to rapidly internalize bacteria into the intestinal mucosa, the suspension with salmonella was administered orally using a probe. Vancomycin was administered to animals at a rate of $50 \mathrm{mg}$ per $\mathrm{kg}$ of body weight, suspensions of microorganisms - in an amount of $15 \mathrm{ml}$ with a concentration of $3 \times 108 \mathrm{CFU} / \mathrm{g}$. As a material for bacteriological studies of the intestinal 
microflora used washes from the ileum of rats. The quantitative and qualitative composition of the wall microbiota in rats by bacteriological method, the statistical analysis of data using the program StatSoft Statistica v12 were conducted. Results. The introduction of vancomycin and S. enteritidis, $S$. typhimurium led to changes in the qualitative and quantitative composition of the intestinal microbiome. The introduction of $S$. enteritidis and $S$. typhimurium, on the background of pre-treatment with vancomycin, caused more pronounced changes: increase of the content of E. coli 65 and 105 times, Enterobacter spp., Klebsiella spp., P. aeruginosa $(\mathrm{p} \leq 0.05)$, and also a sharp decrease in Bacteroides spp. 9 and 10 times, respectively, Proteus spp. 17 times, Peptostreptococcus anaerobius 20 and 9 times, Shigella spp. at 538 and 860 times and Lactobacillus at 17 times. Correction of the microflora of rats of $B$. fragilis leads to a sharp decrease of the number of Salmonella spp., P. aeruginosa, Enterobacter spp., Klebsiella spp., Shigella spp. $(\mathrm{p} \leq 0.05)$, E. coli 538 times, Proteus spp. 322 times, Acinetobacter spp. at 6 and 57 times, Cryptococcus spp. 7-fold, and increase in Bacteroides spp., E. faecalis, E. faecium 10 and 19-fold, Peptostreptococcus anaerobius 7 and 12-fold, Lactobacillus spp. 27 and 40 times, respectively. Conclusions. When B. fragilis was administered to experimental animals treated with $\mathrm{S}$. enteritidis or S. typhimurium on the background of pre-treatment with vancomycin, a change in the quantitative composition of the microbiota in the parietal contents of the small intestine was observed, namely: a decrease in Salmonella spp., E. coli, P. aeruginosa, Proteus spp., Enterobacter spp., Klebsiella spp., As well as an increase in Bacteroides spp., E. faecalis, E. faecium, Lactobacillus spp. and Peptostreptococcus anaerobius. This prove that the introduction of $B$. fragilis can be used in the treatment of inflammatory bowel diseases or diseases with impaired barrier function of the intestine.

\section{References:}

1. Macpherson N. L. Harris Macpherson AJ. Interactions between commensal intestinal bacteria and the immune system. Nature Reviews Immunology. 2004. Vol. 4. P. 478-485.

2. Deplancke B., Gaskins Deplancke H. R. Microbial modulation of innate defense: goblet cells and the intestinal mucus layer. The American Journal of Clinical Nutrition. 2001. Vol. 73. P. 1131-1141.

3. Kau A. L., Ahern P. P., Griffin N. W., Goodman A. L., Gordon J. I. Human nutrition, the gut microbiome and the immune system. Nature. 2011. Vol. 474. P. 327-336. 
4. Stecher B., Hardt W. D. The role of microbiota in infectious disease. Trends Microbiology. 2008. Vol. 16. P. 107-114.

5. Vollaard E. J., Clasener H. A. Colonization resistance. Antimicrobial Agents and Chemotherapy. 1994. Vol. 38. P. 409-414.

6. Stecher B., Hardt W. D. Mechanisms controlling pathogen colonization of the gut. Current Opinion in Microbiology. 2011. Vol. 14. P. 82-91.

7. Monack D. M., Bouley D. M., Falkow S. J. Salmonella typhimurium persists with in macrophages in the mesenteric lymph nodes of chronically infected Nramp1+/+ mice and can be reactivated by IFNgamma neutralization. Experimental Medicine. 2004. Vol. 199. P. 231-241.

8. Jernberg C., Löfmark S., Edlund C., Jansson J. K. Long-term impacts of antibiotic exposure on the human intestinal microbiota. Microbiology. 2010. Vol. 156. P. 3216-3223.

9. Ubeda C., Pamer E.G. Antibiotics, microbiota and immune defense. Trends Immunology. 2012. Vol. 33. P. 459-466.

10. Pérez-Cobas A. E., Artacho A., Knecht H., Ferrús M. L., Friedrichs A., Ott S. J. Differential effects of antibiotic therapy on the structure and function of human gut microbiota. PLoS One. 2013. Vol. 8. P. 201-208.

11. Cho I., Yamanishi S., Cox L., Methé B. A., Zavadil J., Li K. Antibiotics in early life alter the murine colonic microbiome and adiposity. Nature. 2012. Vol. 488. P. 621-626.

12. Zhang Y., Limaye P. B., Renaud H. J., Klaassen C. D. Effect of various antibiotics on modulation of intestinal microbiota and bile acid profile in mice // Toxicology and Applied Pharmacology. 2014. Vol. 277. P. 138-145.

13. Fujimura K. E., Slusher N. A., Cabana M. D., Lynch S. V. Role of the gut microbiota in defining human health. Expert Review of Anti-infective Therapy. 2010. Vol. 8. P. 435-454.

14. Wlodarska M., Willing B., Keeney K. M., Menendez A., Bergstrom K. S., Gill N. Antibiotic treatment alters the colonic mucus layer and predisposes the host to exacerbated Citrobacter rodentium-induced colitis. PubMed. 2011. Vol. 79. P. 1536-1545.

15. Cani P. D., Possemiers S., Van de Wiele T., Guiot Y., Everard A., Rottier O. Changes in gut microbiota control inflammation in obese mice through a mechanism involving GLP-2-driven improvement of gut permeability. PubMed. 2009. Vol. 58. P. 1091-1093. 